Review

\title{
P53 and Sirt1: Routes of metabolism and genome stability
}

\author{
Stefania Gonfloni ${ }^{\mathrm{a}, \mathrm{b}, *}$, Valentina Iannizzotto ${ }^{\mathrm{a}, 1}$, Emiliano Maiani ${ }^{\mathrm{a}, 1,2}$, \\ Giovanna Bellusci ${ }^{a}$, Sarah Ciccone ${ }^{a}$, Marc Diederich ${ }^{c}$ \\ a Department of Biology, University of Rome "Tor Vergata", Via della Ricerca Scientifica, 00133 Rome, Italy \\ ${ }^{\mathrm{b}}$ Laboratoire de Biologie Moléculaire et Cellulaire du Cancer, Kirchberg Hospital, 9 Rue Edward Steichen, 2540 Luxembourg, Luxembourg

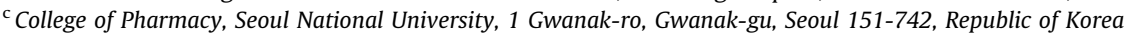

\section{A R T I C L E I N F O}

\section{Article history:}

Received 28 June 2014

Received in revised form 28 August 2014

Accepted 29 August 2014

Available online $\mathrm{xxx}$

\section{Keywords:}

p53

SIRT1

Metabolism

Tumor suppression

Genome integrity

\begin{abstract}
A B S T R A C T
The tumor suppressor $\mathrm{p} 53$ is a transcription factor that regulates key processes. But, the outcomes of the p53 response go beyond its role as a nuclear transcription factor. Sirtuin (SIRT1) regulates p53 functions as transcription factor. At the same time, SIRT1 protects the genome under stress conditions. The link between p53 and SIRT1 responses is unique. Both regulate metabolism, stress signaling, cell survival, cell cycle control and genome stability. Recent studies have proposed cancer as a metabolic disease. This is due to the switch from aerobic to anaerobic metabolism during tumor development. Yet, the complex molecular circuits (in and out of the nucleus) of tumor progression remain elusive. In this review, we will focus on the interplay between p53 and SIRT1. We will discuss their roles as nodes for possible therapeutic intervention.
\end{abstract}

(c) 2014 Elsevier Inc. All rights reserved.

\section{Contents}

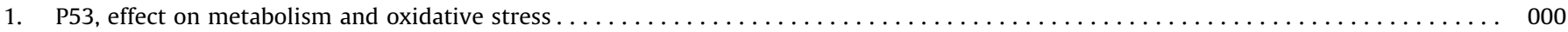

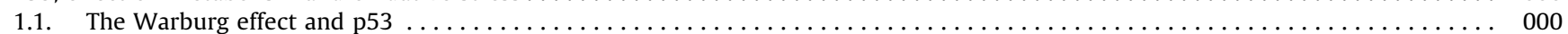

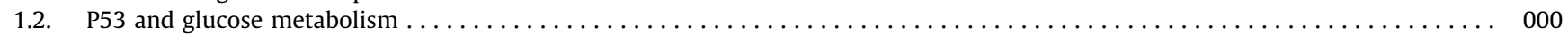

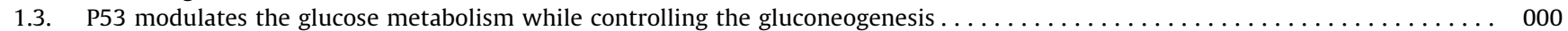

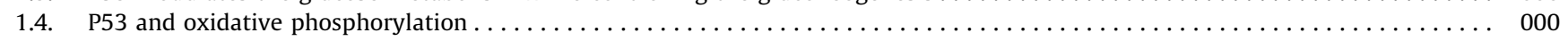

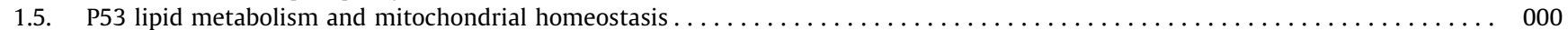

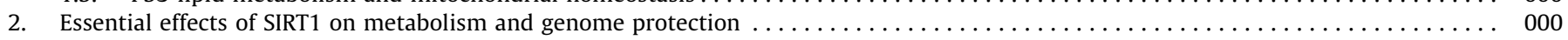

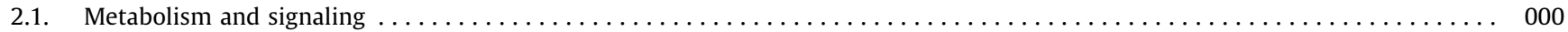

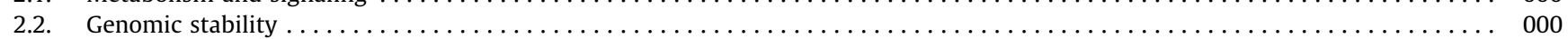

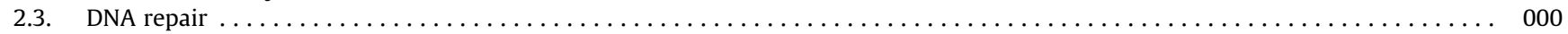

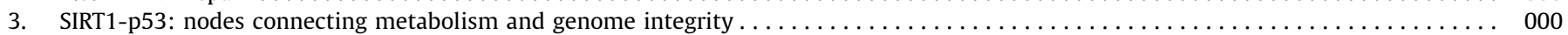

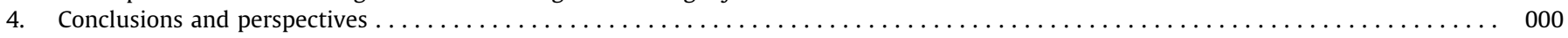

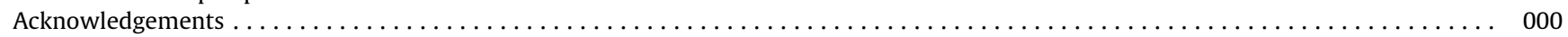

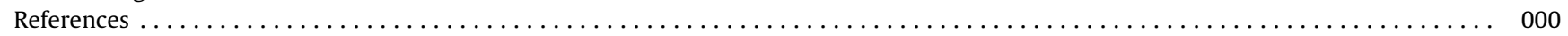

\footnotetext{
* Corresponding author.

E-mail addresses: Stefania.Gonfloni@uniroma2.it, Stefania.Gonfloni@lbmcc.lu (S. Gonfloni).

${ }^{1}$ These authors contribute equally to this work.

2 Present address: Cell Stress and Survival Unit, Danish Cancer Society Research Center, Strandboulevarden 49, 2100 Copenhagen, Denmark.
}

\section{P53, effect on metabolism and oxidative stress}

Metabolic changes take place with tumor progression. Cancer cells often rewire their metabolic pathways to promote fast growing and genomic instability. The best-understood function of p53 is its central role as tumor suppressor. Emerging evidence indicates a role of p53 in monitoring/modulating cell metabolism [1]. Cell fate depends either by p53 transcription-dependent or independent responses within mitochondria. P53 regulates many 
proteins required for metabolism and reactive oxygen species (ROS) production. Besides, p53 controls redox signaling, through the modulated expression of pro-and anti-oxidant proteins [2].

\subsection{The Warburg effect and $p 53$}

Transformed cells get a series of features to proliferate fast and to escape from programmed cell death. In 1927 Otto Warburg demonstrated that tumor progression takes place together with metabolic changes [3]. Warburg observed that cancer cells shift from oxidative phosphorylation (OXPHOS) to glycolysis. This occurs even in presence of oxygen. This process is now recalled as the "Warburg effect". Glycolysis takes part in the cytoplasm. It leads to the NADH and ATP production through conversion of glucose into pyruvate. Oxidative phosphorylation generates ATP into mitochondria. In resting conditions OXPHOS is predominant and more efficient. Even if, cells with high rates of proliferation tend to switch to glycolysis. Cancer cells make use of the pentose phosphate pathway (PPP), which originates from a bypass in the glycolysis [4]. PPP is necessary for lipid and nucleic acid synthesis and represents an important source of NADPH. NADPH is also necessary for the production of glutathione (GSH), the major intracellular antioxidant. Thus, the PPP leads to proliferate fast and to protection against oxidative stress [5].

P53 is the most important obstacle for tumor progression. The p53 homologue is present in unicellular organisms. This indicates that the tumor suppression may not be p53's original function [6]. Besides DNA damage and oncogene activation, nutrient flow changes activate p53 [7]. Recent studies show that p53 controls the metabolic switch between glycolysis and oxidative phosphorylation.

\subsection{P53 and glucose metabolism}

P53 inhibits glycolysis by acting at different levels. It has been demonstrated that $\mathrm{p} 53$ decreases glucose import by reducing the expression of GLUT1 and GLUT4 glucose transporters [8]. P53, through the inhibition of IKK, also regulates the GLUT3 expression [9]. Bensaad and co-workers demonstrated that p53 reduces glycolysis by promoting TIGAR expression [5]. TIGAR dephosphorylates fructose-2,6-bisphosphate to fructose-6-phosphate and blocks the breakdown of glucose into pyruvate. This in turn promotes the switch to the oxidative pentose phosphate pathway (ox-PPP). The latter leads to NADPH production and to a more effective protection against oxidative stress. P53-independent TIGAR accumulation is a hallmark of several tumors. Yet, the scenario is complex since p53 inhibits the glucose-6-phosphate dehydrogenase (G6PDH) expression. G6PDH takes part in the first and limiting step of the ox-PPP [4]. The mechanism by which p53 inhibits G6PDH represents a way to block ox-PPP in case of accumulation of TIGAR in cancer cells. TAp73, a member of the p53 family, induces the expression of G6PDH genes and leads to cell proliferation [10]. In mouse embryonic fibroblasts, p53 inhibits glycolysis. In these cells, P53 reduces the protein levels of the glycolytic enzyme phosphoglycerate mutase (PGM). PGM converts 3-phosphoglycerate into 2-phosphoglycerate [11]. However, p53 promotes glycolysis in muscle by inducing the expression of PGM $M$ isoform [12] and of hexokinase II (HK2) [13]. The latter converts glucose into glucose-6-phosphate in the first step of glycolysis. This indicates that the effects of p53 on glycolysis are remarkable and tissue-specific.

\subsection{P53 modulates the glucose metabolism while controlling the gluconeogenesis}

Gluconeogenesis produces glucose and is essential for tumor cell growth. P53 represses the gluconeogenesis by promoting the expression of histone deacetilase sirtuin 6 (SIRT6). SIRT6 deacetylates forkhead box protein 01 (FOXO1). In turn, this represses the expression of glucose-6-phosphatase (G6PC) and of phosphoenolpyruvate carboxykinase (PCK1). Both enzymes are rate-limiting proteins for gluconeogenesis [14].

\subsection{P53 and oxidative phosphorylation}

While repressing glycolysis, p53 promotes oxidative phosphorylation at distinct levels. In presence of oxygen, the pyruvate derived from glycolysis is converted in Acetyl coenzyme A (AcetylCoA). The latter takes part in the tricarboxylic acid (TCA) cycle. In TCA cycle, ATP is generated through oxidative phosphorylation. Besides, TCA cycle provides precursors for anabolic pathways, so supporting cell growth and proliferation. P53-deficient cells produce less ATP from the TCA cycle compared to control cells [15]. In detail, p53 promotes TCA cycle by reducing the pyruvate dehydrogenase kinase 2 (PDK2) expression. PDK2 inactivates the pyruvate dehydrogenase complex (PCD). In turn this promotes the conversion of pyruvate into lactate instead of Acetyl-CoA[16].

P53 enhances gene transcription of mitochondrial components, including subunit 1 of cytochrome c oxidase (COI) [17], and cytochrome c oxidase 2 (SCO2). SCO2 in turn regulates the subunit 1 of complex IV [15]. Besides, p53 induces the expression of the mitochondrial apoptosis-inducing factor (AIF) [18]. AIF acts as $\mathrm{NADH} / \mathrm{NADPH}$ oxidase. AIF is also essential for the proper functioning of complex I.

P53 reduces the expression of the TCA cycle-associated malic enzymes ME1 and ME2. These enzymes are important for NADPH production, lipogenesis and glutamine metabolism. Thus, P53 regulates both the progression of biosynthetic pathways and the antioxidant response [19]. Besides, p53 enhances OXPHOS by promoting the transcription of glutaminase 2 (GLS2) [20,21]. GLS2 stimulates the production of glutamate and $\alpha$-ketoglutarate. The latter is a key component of the TCA cycle involved in ATP production and the antioxidant response.

\subsection{P53 lipid metabolism and mitochondrial homeostasis}

Besides alterations in glycolysis and oxidative phosphorylation, cancer cells show a deregulated lipid metabolism. In particular, cancer cells synthesize fatty acids. The fatty acids are the main reserve of lipids. Lipids are necessary for membrane formation and signaling transduction [22]. Breast, colon and prostate cancer cells [23-25] have high levels of fatty acids synthases (FASN). This observation supports the role of FASN in tumorigenesis. In line with this, FASN inhibition counteracts cellular transformation (reviewed in [26]). FASN deficiency counteracts cancer growth in cells with activated PI3K signaling. Yet, blocking FASN in K-Rasdriven cancer cells has no effect on proliferation [27].

P53 attenuates the fatty acids synthesis, repressing the expression of the transcriptional regulator SREBP-1 (sterol regulatory element-binding protein $1 \mathrm{c}$ ). The latter promotes the expression of triglyceride synthesis and lipogenic enzymes [28]. Thus, p53 inhibits the expression of FASN and (ATP citrate lyase) ACLY genes. Thereby, p53 may repress tumor proliferation while inhibiting the fatty acids synthesis. The fatty acid oxidation leads to the formation of Acetyl-CoA, which takes part in the TCA cycle, producing ATP, NADPH and FADPH. As mentioned above, TCA cycle is active in resting conditions. While, NADPH is necessary for the cellular protection against oxidative stress. Upon glucose deprivation, p53 induces the expression of Lpin1. This in turn stimulates the fatty acids oxidation [29]. Lpin1 is a nuclear transcriptional co-activator. Following glucose starvation Lpin1 induces the expression of genes involved in fatty acids oxidation. But, Lpin1 inhibits the fatty acid oxidation at high glucose. 


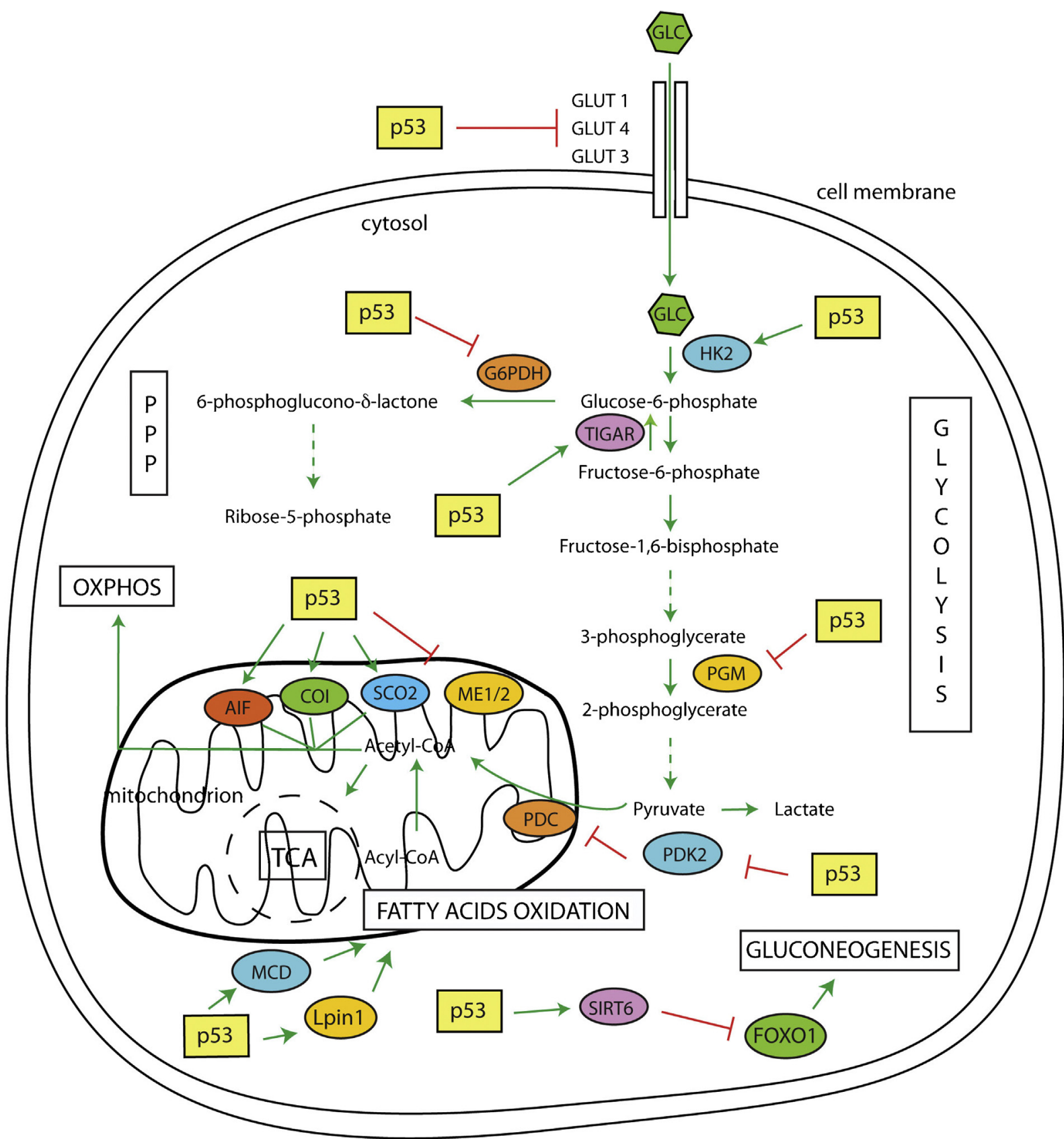

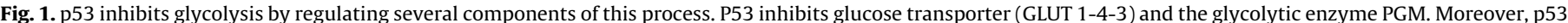

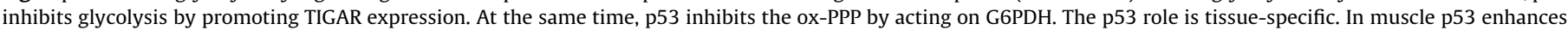

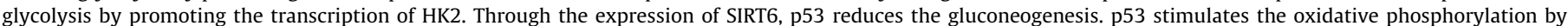

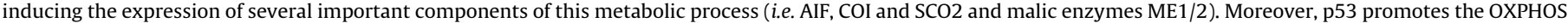

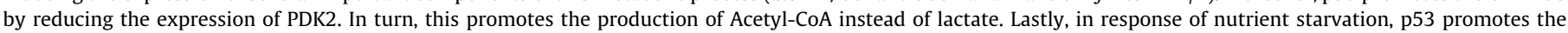
expression of Lpin1 and MCD, stimulating the conversion of Acyl-CoA to Acetyl-CoA. In this way p53 stimulates the oxidative phosphorylation instead of glycolysis.

In mice, P53 controls the hepatic fatty acid oxidation in response to fasting [30]. P53 induces the transcription of malonylCoA decarboxylase (MCD). MCD stimulates the malonyl-CoA turnover and increases carnitine palmitoyl transferase activity. This leads to mitochondrial fatty acid uptake (Fig. 1).

In brief, P53 enhances the oxidative respiration in cells by promoting the fatty acid oxidation. In this manner, p53 may counteract the Warburg effect.

Mitochondrial DNA (mtDNA) contains genes of enzymatic complexes involved in OXPHOS. P53 protects mitochondrial DNA from mutations, promoting the transcription of the ribonucleotide reductase p53R2. Besides, p53 preserves mtDNA integrity and regulates the mtDNA copy number [31]. Thereby, p53 promotes the oxidative phosphorylation while ensuring mitochondrial DNA protection.

Yet, it remains an open question whether (and to what extent) the metabolic status may contribute to genomic maintenance. Recent evidence suggests that genomic stability requires cooperation of p53 and SIRT1.

\section{Essential effects of SIRT1 on metabolism and genome protection}

Sirtuins are a family of class III deacetylases ( $\mathrm{NAD}^{+}$-dependent). These enzymes control many cellular pathways, including metabolism, stress and genome stability. 
Table 1

Sirt substrates.

\begin{tabular}{lll}
\hline Substrates & Cellular function & References \\
\hline H4 & Histones & PMID: 17694090 \\
H3 & & PMID: 19411844 \\
H1 & Histone-modifying enzymes & PMID: 15469825 \\
SUV39H1 & & PMID: 21504832 \\
PCAF & & PMID: 19188449 \\
p300 & Pranscription factors & PMID: 15632193 \\
TIP60 & & PMID: 22586264 \\
p53 & & PMID: 11672523 \\
E2F1 & & PMID: 16892051 \\
NFkB & & PMID: 15152190 \\
P73 & & PMID: 16998810 \\
FOXO 1 & & PMID: 25009184 \\
FOXO 3a & PMID: 14976264 \\
Myc & & PMID: 21807113 \\
HIF-1 $\alpha /$ HIF2- $\alpha$ & & PMID: 24423936 \\
ATM & & PMID: 23852118 \\
AMPK & Cell signaling components & PMID: 19276888 \\
AKT & & PMID: 24436432 \\
Ku70 & & PMID: 23247197 \\
WRN & & PMID: 18203716 \\
Rad51 & DNA repair modulators & PMID: 20097625 \\
NBS1 & & PMID: 17612497 \\
APE1 & & PMID: 19934257 \\
BRCA1 & & PMID: 18851829 \\
PGC-1a & PMID: 15744310 \\
CLOCK & & PMID: 24442997 \\
PER2 & & PMID: 18662546 \\
PPARg & & PMID: 15175761 \\
\hline & & \\
\hline & &
\end{tabular}

The basic function of Sirtuins links chromatin dynamics/gene expression to environmental stimuli. At the same time, Sirtuins ensure genome protection [32]. Genome integrity relies on chromatin structure and DNA repair mechanisms. Chromatin and DNA repair depend by deacetylation of histones (and of other chromatin-associated factors) [33,34]. Sirtuins were discovered in yeast (Silent Information Regulator, Sir2). They were genes necessary for the repression of silent-mating type loci [35]. The mammalian family of Sirtuins comprises of seven proteins, SIRT1SIRT7. These enzymes are ubiquitously expressed. SIRT1 is the best-studied gene and the largest in sequence. $\mathrm{NAD}^{+}$, allosteric modulators, nucleo-cytoplasm shuttling, transcription regulators activate SIRT1 [36]. The mammalian Sirtuins are present in distinct subcellular compartments. Some Sirtuins are present into mitochondria. This implies a role of Sirtuins for metabolism and for mitochondria homeostasis [37].

\subsection{Metabolism and signaling}

Metabolism and signaling pathways are interconnected [38]. Cancer cells exploit the signalling-dependent regulation of metabolism to fuel macromolecular biosynthesis. This supports their fast growth [39-41]. Nutrient-sensing molecules are the AMP-activated kinases (AMPK) and the mammalian target of rapamycin complex 1 (mTORC1). These enzymes are key regulators of the metabolic status of the cells. Besides, metabolites generate posttranslational modifications (PTMs) [42].

Cytosolic and nuclear Acetyl-CoA levels modulate PTMs, gene expression, signaling cascades and metabolism [38]. Two opposite enzymes as acetyltransferases and deacetylases regulate acetylation. The latter is a PTM induced by the metabolite Acetyl-CoA $[43,44]$. Intracellular metabolic conditions control the activity of these two classes of enzymes. SIRT1 deacetylase depends on NAD ${ }^{+}$ as a cofactor. Besides $\mathrm{NAD}^{+}, \mathrm{NAD}^{+}$biosynthetic enzymes (as Nicotinamide phosphoribosyltransferase - Nampt) promote SIRT1 deacetylase activity.

Proteomic studies indicated that acetylation (and Acetyl-CoA production) regulates several signaling events. Emerging concepts suggest that acetylation is like a "sensor" of Acetyl-CoA availability within cells. ATP-citrate lyase (ACL) and Acetyl-CoA synthetase 1 (ACECS1) are essential enzymes for Acetyl-CoA production. ACECS1 is deacetylated (and activated) by SIRT1 under nutrientlimited conditions [45]. Acetylation of ACECS1 may serve as a regulatory/inhibitory modification in response to metabolic changes. Many enzymes involved in metabolism are also acetylated $[46,47]$. An acetyl-mimic mutant of pyruvate kinase (PKM2) promotes tumor xenografs growth [48].

Besides, Acetyl-CoA production enhances histone acetylation and so gene transcription [49]. Nutrient availability, metabolic conditions and posttranslational modifications of the enzymes control Acetyl-CoA production. Phosphorylation of ACL by AKT and by PKA increases ACL activity [50-52]. Cancer cells have the phosphoinositide 3-kinase (PI3K)-AKT pathway activated. AcetylCoA production is persistent, providing a continuous signal to promote growth [38]. PTMs of deacetylases are linked to AcetylCoA production. SIRT1 modifications exert either stimulatory or inhibitory effects on SIRT1 activity [53]. Different kinases/ modifiers and diverse multi-site regulatory mechanisms control SIRT1 activity [54]. In response to oxidative stress, c-Jun Nterminal kinase (JNK) phosphorylates SIRT1 at three sites [55]. These modifications increase SIRT1 activity. In contrast, mTOR phosphorylates SIRT1 at a single site (S47) in response to oxidative stress. This modification inhibits SIRT1 activity. AMPK targets human SIRT1 at T344. This modification reduces SIRT1 capability to deacetylate p53 [56,57]. Other kinases as CK2, DYRK1 and DYRK3 phosphorylate SIRT1 in different sites. These modifications in turn stimulate SIRT1 activity [58-60]. In contrast, CyclinB/ Cdk1 (a cell cycle dependent kinase) phosphorylates SIRT1 at T530 and S540. Both modifications inhibit SIRT1 activity [61].

The first discovered non-histone substrate of SIRT1 is p53. P53 plays a central role in SIRT1-mediated senescence and in tumor progression [62]. SIRT1 represses p53-dependent transactivation in tumors or in mouse embryonic fibroblasts [63]. Yet, p53 binding to SIRT1 promoter represses SIRT1 transcription [62]. Thus, p53 provides a feedback circuit either to regulate SIRT1 expression and the p53 response. Low nutrient conditions increase NAD/NADH ratio, promoting SIRT1 activity. PARP1 activity leads to NAD+ depletion, limiting SIRT1 activity [64]. Moreover, SIRT1 induces gene silencing through H3K9 deacetylation of target gene promoters (Fig. 2).

SIRT1 reduces the p53-dependent apoptosis induced by stress [65,66]. SIRT1-deficient mice show p53 hyperacetylation following DNA damage. They also show an increased ionizing radiationinduced apoptosis in thymocytes [66]. Other studies report a modest effect of SIRT1 on the p53-mediated responses $[67,68]$. These opposite results imply the existence of other mechanisms by which SIRT1 exerts its effect on p53. Convincing evidence indicates that SIRT1 regulates the p53 subcellular localization. Likely, SIRT1 prevents p53 nuclear translocation. This in turn promotes cytosolic accumulation of $\mathrm{p} 53$ and its passage to mitochondria. In brief, SIRT1 blocks p53 transcription-dependent apoptosis. At the same time SIRT1 increases p53-mediated (transcription-independent) apoptosis [62].

SIRT1 was first considered as a potential tumor promoter, repressing p53. Yet, recent results indicated that Sirt1 acts as tumor suppressor by facilitating mitochondria-dependent apoptotic response.

SIRT1 deacetylates different histones $(\mathrm{H} 4, \mathrm{H} 3$, and $\mathrm{H} 1)$ and also many non-histone proteins. SIRT1 substrates take part in diverse cellular responses $[69,70]$. SIRT1 substrates can be 1) histonemodifying enzymes such as SUV39H1, PCAF, p300 and TIP60; 2) transcription factors regulating cell cycle progression/survival under various stress conditions ( $\mathrm{p} 53$, nuclear factor (NF)- $\kappa B$, p73, forkhead transcription factors FOXO1, FOXO3a, Myc, 


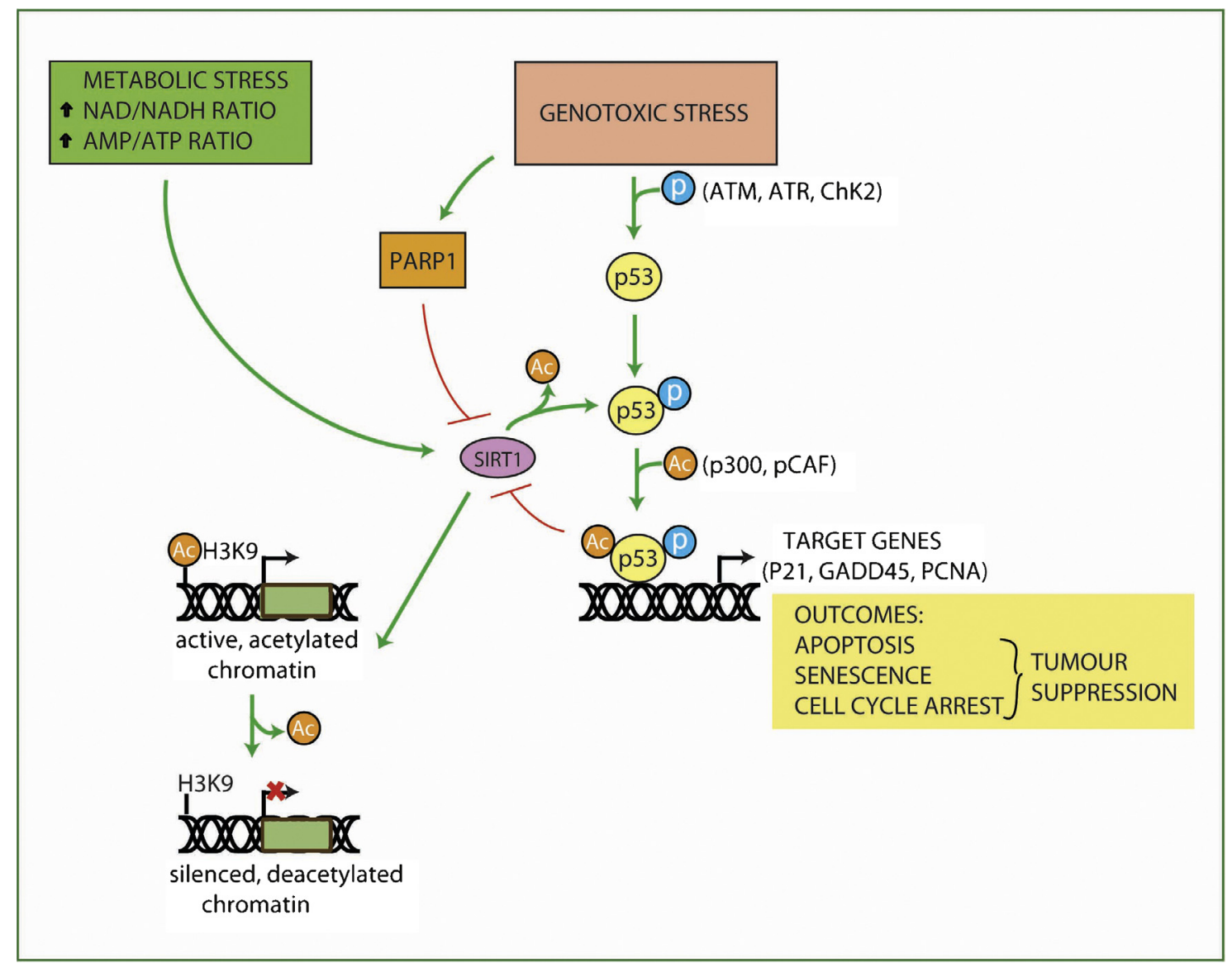

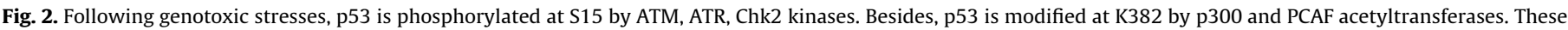

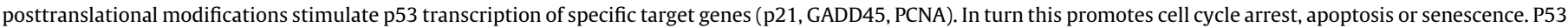

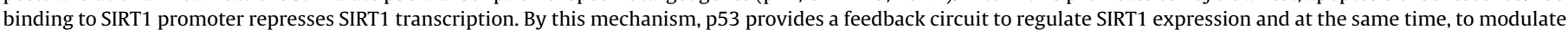

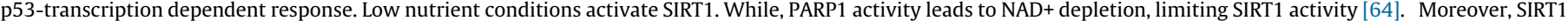
induces gene silencing through H3K9 deacetylation of target gene promoters.

hypoxia-inducible transcription factors (HIF)-1 $\alpha$ and HIF2- $\alpha$ ); 3 ) cell signaling components (modifiers, enzymes) AKT; 4) DNA repair modulators such as Ku70, WRN, NBS1, APE1; 5) regulators of metabolism, circadian clock including PGC-1 $\alpha$, PER2 [71,72] (see Table 1).

Mouse models support the maintenance of genomic stability as the fundamental role of SIRT1 [32]. Yet, SIRT1 modulates the regulatory signaling circuits of cell homeostasis. SIRT1 may deal with oxidative stress, aging, metabolism, and genome protection.

To date, the identification of Sirtuin-regulated signaling targets remains incomplete. Reversal of the acetylation affects transcription factors [66] and their nuclear localization. Yet, removal of acetylation may induce protein breakdown [73].

Acetylation of the mitogen-activated protein kinase kinase-1 (MEK1) enhances its kinase activity. Treatment with Sirtuin inhibitors or siRNA silencing of SIRT1 or SIRT2, enhances MEK1 acetylation. This modification causes the consequent phosphorylation of extracellular signal-regulated kinase (ERK). An acetylmimic mutant of MEK1 promotes inappropriate growth properties. This indicates that acetylation of MEK1 may exert an oncogenic potential [74]. It is of interest to point out that SIRT2 is a new AKT adaptor required for optimal AKT activity. Constitutive SIRT2 binding to AKT is dependent on phosphorylation at T101 by AMP-activated kinase. Pharmacological inhibition or genetic down-regulation of SIRT2 inhibits AKT activation in stimulated cells. Of note, overexpression of SIRT2 enhances AKTmediated pathway activation. In cells with constitutive PI3K activation, AKT interacts with a nuclear Sirtuin, (SIRT1). But, inhibition of PI3K induces SIRT1 dissociation and increased association with SIRT2. This supports the use of SIRT1 and SIRT2 inhibitors in the treatment of tumor cells with an increased PI3K activity [75].

Reversible acetylation controls the subcellular localization of many other signaling factors/components. Besides, it regulates the silencing of gene expression. SIRT1 promotes nuclear retention of High-mobility group (HMG) B1. SIRT1 modulates damage signaling initiated by HMGB1 secretion upon stress [76]. SIRT1 reduces the expression of survivin, through $\mathrm{H} 3 \mathrm{~K} 9$ deacetylation on its promoter [77].

SIRT1 may promote and/or suppress tumorigenesis $[32,78,79]$. On one hand, SIRT1-deficiency may help tumor progression, promoting genomic instability. Yet, tumor cells tend to need of Sirtuins to proliferate, to repair with low fidelity and to evolve [78]. The bifurcated role of SIRT1 in tumorigenesis is due to its peculiar role in genome protection [78].

\subsection{Genomic stability}

SIRT1 acts on the genome through various mechanisms and at different levels. SIRT1 regulates chromatin modifications, facilitating DNA repair. Besides, SIRT1 connects energy and metabolic flows to chromatin dynamics and gene expression.

SIRT1 facilitates both constitutive heterochromatin $(\mathrm{CH})$ and facultative heterochromatin (FH) formation. SIRT1 enhances methyl-transferases activity. This promotes the methylation of CpG islands (reviewed in [79]). 
SIRT1 induces epigenetic silencing of target genes [80,81]. SIRT1 modifies histone (H4K16Ac, H3K9Ac, H1K26Ac) and chromatin factors (as the histone methyl-transferase SUV39H1). Loss of SIRT1 prevents both the spreading of heterochromatin marks (H3K79me3) and localization of heterochromatin protein 1 (HP1) [82]. The interplay between SIRT1 and SUV39H1 is mediated by the E3 ubiquitin ligase MDM2. SIRT1 inhibits SUV39H1 polyubiquitination by MDM2 and so increases SUV39H1[83]. High levels of SUV39H1 in vivo enhance its turnover in pericentromeric heterochromatin regions. This ensures genome integrity. SIRT1 may generate aberrant methylation of the $\mathrm{CpG}$ islands in promoters following DNA damage. This promotes heritable gene silencing [84]. SIRT1-mediated gene silencing may affect both tumor promoting and tumor suppressor genes. SIRT1 inhibition reactivates silenced tumor suppressor genes in cancer cell. This occurs without loss of promoter hypermethylation [85].

\subsection{DNA repair}

SIRT1 takes part in DNA signaling and repair at different levels. For the repair of singlestrand breaks (SSBs), SIRT1 controls the nucleotide excision repair (NER) pathway. SIRT1 interacts with XPA (xeroderma pigmentosum complementation group A) [86]. SIRT1 enhances XPC (xeroderma pigmentosum complementation group C) expression. SIRT1 prevents AKT-mediated nuclear localization of XPC transcriptional repressors. Inhibition of SIRT1 affects XPC transcription, impairing NER function [87]. Several DNA repair factors are also substrates of SIRT1, among them X-ray repair crosscomplementing gene 4 protein (XRCC4p) [88]; Ku70 [89]; Werner's Syndrome protein (WRN)[90]. SIRT1 binds to and modifies Nijmegen breakage syndrome protein (NBS1). NBS1 is a regulatory component of the MRE11-Rad51-NBS (MRN) nuclear complex [91,92]. MRN complex works as a "sensor" in the early stages of double strand break (DSB) signaling. MRN promotes the formation of $\gamma$-H2AX foci upon $\gamma$-irradiation [93]. SIRT1 recruitment to DSBs depends on ATM-mediated signaling (i.e. $\gamma$-H2AX phosphorylation) [94]. SIRT1 interacts with (and modifies) two members of the MYST acetyltransferase family (hMOF and TIP60). Both enzymes are necessary for cell growth, DNA repair and apoptosis [95-97]. SIRT1 inhibits their activity by promoting their ubiquitin-mediated degradation. Following DNA breaks, SIRT1 binding is reduced and hMOF and TIP60 take part in the damage response [97-99]. SIRT1 is also connected to the non-homologous end joining (NHEJ) repair pathway, through the protein Ku70. Deacetylation of Ku70 by SIRT1 prevents Ku70 translocation. At the same time, SIRT1 promotes Ku70-dependent DNA repair [100-102].

\section{SIRT1-p53: nodes connecting metabolism and genome integrity}

Mouse models support a role of SIRT1 for genome protection $[66,93,103,104]$. SIRT1 regulates p53 acetylation. SIRT1-deficient thymocytes exhibit p53 hyperacetylation [66]. Double knockout mice show that genomic integrity needs cooperation between p53 and SIRT1 [93].

In mice, evidence shows that p53 tumor suppressor function relies on p53 ability to modulate metabolism [105]. Besides, the p53 family members p63 and p73 are emerging as critical components in the regulation of metabolism [106,107]. This implies that the metabolic functions of p63/p73 may also contribute to tumor suppression. Yet, these effects remain elusive in particular with tumors expressing mutant forms of p53 [108].

SIRT1 modulates both p53 transcription-dependent and p53independent apoptosis. The latter is initiated through cytochrome $c$ release from the mitochondria. Emerging evidence indicates that SIRT1 prevents p53 nuclear translocation. SIRT1 redirects p53 from the cytosol to the mitochondria in response to increased ROS. Likely, the biological effect of p53 enrichment into mitochondria induces transcription-independent apoptosis. In this way, oxidative stress modulates cell fate through SIRT1-mediated p53 deacetylation (reviewed in[62]).

DNA Damage stress Response (DDR) relies on cell cycle checkpoints for repairing DNA lesions. Failure to balancing of DDR response promotes ageing and cancer. How are cellular metabolism and DDR interconnected? Besides, the roles of p53 and SIRT1 remain still undefined.

The chemotherapeutic drug cisplatin, causes oxidative stress and various types of DNA alterations. Stechow and co-workers perform metabolic profiling by mass spectrometry (MS) of embryonic stem (ES) cells. ES cells are treated for different time periods with cisplatin. MS and transcriptomics analyses of cisplatin-treated ES-cells show that cisplatin alters the metabolic pathways. Yet, several metabolic enzymes induced by cisplatin are also p53 target genes. These results show that metabolic pathways are interconnected with DNA damage response. It is of interest that p53 plays a central role for both processes [109].

Emerging evidence indicates that metabolic changes have a direct effect on chromatin and DNA repair. Several chromatinmodifying enzymes, involved in DNA repair, are regulated by metabolic cofactors. Thus, alterations of energy and metabolic flows have profound consequences on genome integrity.

Chromatin plays an essential role in DNA repair pathways (nonhomologous end-joining (NHEJ) or homologous recombination (HR)). Many histone modifications are necessary to assembly signaling-platforms for repair mechanisms. Histone modifications (including phosphorylation, acetylation, methylation, ubiquitylation) regulate DSB repair [110-114]. Enzymes, involved in histone modifications, need metabolites as cofactors or substrates for their activity. Metabolic cofactors/substrates including Acetyl-CoA (involved in Acetyl-CoA-dependent histone acetylation) and $\mathrm{NAD}^{+}$modulate histone modifications. $\mathrm{NAD}^{+}$is essential for $\mathrm{NAD}^{+}$-dependent deacetylation or $\mathrm{NAD}^{+}$-dependent polyADPribosylation of chromatin. Besides, $S$-adenosyl-L-methionine (SAM) and FAD $/ \alpha$-ketoglutarate induce methylation and demethylation of histones and/or DNA. Metabolic modifications may contribute to DNA double-strand (DSBs) repair defects [115]. At what extent do the metabolic changes interfere with chromatindirected repair processes? It remains an open question. These studies represent an exciting area of investigation.

\section{Conclusions and perspectives}

Cancer is a complex disease driven by epigenetic changes, genetic modifications in oncogenes and tumor suppressors. Yet, alterations in metabolism have profound effects on the cell growth/survival. Cell homeostasis requires fine-tuning of energy and metabolic flows with genome stability. P53 and SIRT1 are nodes regulating metabolism, DNA repair and senescence.

Metabolic p53 responses are important for maintaining cell homeostasis and for preventing tumor development. Metabolic and other stress signals activate p53 [116]. Recently, other authors reviewed the cytosolic functions of p53 [117]. P53 regulates metabolism and induces senescence. This represents a paradigm for targeting of metabolic alterations associated with cancer [118].

Recent evidence indicates a dual effect of SIRT1 on cancer. SIRT1 enhances cell survival, allowing indefinite cell division. At the same time, SIRT1 promotes genome stability under stress conditions [79].

DNA repair needs dynamic chromatin remodeling, re-assembly of nucleosome, histone variant exchanges, signaling response. Metabolite-associated changes at chromatin may promote malignant transformation through deregulation of DNA repair [115]. How do metabolic changes interfere with chromatin-directed 
repair processes? It remains elusive. The in-depth understanding of metabolite effects on DNA repair will help the development of combined targeted therapies to eradicate cancer.

\section{Acknowledgements}

We acknowledge support from AIRC (Italian Association for Cancer Research, IG grant 2011 No. 11344) to SG. Research in M.D.'s lab is supported by the "Recherche Cancer et Sang", the "Recherches Scientifiques Luxembourg association, the "Een Haerz fir kriibskrank Kanner" association, the Action Lions "Vaincre le Cancer" association and by Télévie Luxembourg. MD is supported by the National Research Foundation of Korea (NRF) grant for the Global Core Research Center (GCRC) funded by the Korea Government, Ministry of Science, ICT \& Future Planning (MSIP) (No. 2011-0030001).

\section{References}

[1] Vousden $\mathrm{KH}$, Prives C. Blinded by the light: the growing complexity of p53. Cell 2009;137:413-31.

[2] Wang DB, Kinoshita C, Kinoshita Y, Morrison RS. p53 and mitochondrial function in neurons. Biochimica et Biophysica Acta 2014;1842(8):1186-97. http://dx.doi.org/10.1016/j.bbadis.2013.12.015.

[3] Warburg O, Wind F, Negelein E. The metabolism of tumors in the body. The Journal of General Physiology 1927;8:519-30.

[4] Jiang P, Du W, Wang X, Mancuso A, Gao X, Wu M, et al. p53 regulates biosynthesis through direct inactivation of glucose-6-phosphate dehydrogenase. Nature Cell Biology 2011:13:310-6.

[5] Bensaad K, Tsuruta A, Selak MA, Vidal MN, Nakano K, Bartrons R, et al. TIGAR, a p53-inducible regulator of glycolysis and apoptosis. Cell 2006;126:107-20.

[6] Lu WJ, Amatruda JF. Abrams JM. p53 ancestry: gazing through an evolutionary lens. Nature Reviews. Cancer 2009;9:758-62.

[7] Maddocks OD, Vousden KH. Metabolic regulation by p53. Journal of Molecular Medicine (Berlin Germany) 2011;89:237-45.

[8] Schwartzenberg-Bar-Yoseph F, Armoni M, Karnieli E. The tumor suppressor p53 down-regulates glucose transporters GLUT1 and GLUT4 gene expression. Cancer Research 2004;64:2627-33

[9] Kawauchi K, Araki K, Tobiume K, Tanaka N. p53 regulates glucose metabolism through an IKK-NF-kappaB pathway and inhibits cell transformation. Nature Cell Biology 2008;10:611-8

[10] Du W, Jiang P, Mancuso A, Stonestrom A, Brewer MD, Minn AJ, et al. TAp73 enhances the pentose phosphate pathway and supports cell proliferation. Nature Cell Biology 2013;15:991-1000.

[11] Kondoh H, Lleonart ME, Gil J, Wang J, Degan P, Peters G, et al. Glycolytic enzymes can modulate cellular life span. Cancer Research 2005:65:177-85.

[12] Ruiz-Lozano P, Hixon ML, Wagner MW, Flores AI, Ikawa S, Baldwin Jr AS, et al p53 is a transcriptional activator of the muscle-specific phosphoglycerate mutase gene and contributes in vivo to the control of its cardiac expression. Cell Growth \& Differentiation: The Molecular Biology Journal of the American Association for Cancer Research 1999;10:295-306.

[13] Mathupala SP, Ko YH, Pedersen PL. Hexokinase II: cancer's double-edged sword acting as both facilitator and gatekeeper of malignancy when bound to mitochondria. Oncogene 2006;25:4777-86.

[14] Zhang P, Tu B, Wang H, Cao Z, Tang M, Zhang C, et al. Tumor suppressor p53 cooperates with SIRT6 to regulate gluconeogenesis by promoting FoxO1 nuclear exclusion. Proceedings of the National Academy of Sciences of the United States of America 2014;111:10684-89.

[15] Matoba S, Kang JG, Patino WD, Wragg A, Boehm M, Gavrilova O, et al. p53 regulates mitochondrial respiration. Science 2006:312:1650-3.

[16] Contractor T, Harris CR. p53 negatively regulates transcription of the pyruvate dehydrogenase kinase Pdk2. Cancer Research 2012;72:560-7.

[17] Okamura S, Ng CC, Koyama K, Takei Y, Arakawa H, Monden M, et al. Identification of seven genes regulated by wild-type p53 in a colon cancer cell line carrying a well-controlled wild-type p53 expression system. Oncology Research 1999;11:281-5.

[18] Vahsen N, Cande C, Briere JJ, Benit P. Joza N, Larochette N, et al. AIF deficiency compromises oxidative phosphorylation. The EMBO Journal 2004;23:4679-89.

[19] Jiang P, Du W, Mancuso A, Wellen KE, Yang X. Reciprocal regulation of p53 and malic enzymes modulates metabolism and senescence. Nature 2013:493:689-93.

[20] Hu W, Zhang C, Wu R, Sun Y, Levine A, Feng Z. Glutaminase 2, a novel p53 target gene regulating energy metabolism and antioxidant function. Proceedings of the National Academy of Sciences of the United States of America 2010;107:7455-60.

[21] Suzuki S, Tanaka T, Poyurovsky MV, Nagano H, Mayama T, Ohkubo S, et al Phosphate-activated glutaminase (GLS2), a p53-inducible regulator of glutamine metabolism and reactive oxygen species. Proceedings of the Nationa Academy of Sciences of the United States of America 2010;107:7461-6.
[22] Santos CR, Schulze A. Lipid metabolism in cancer. The FEBS Journal 2012;279: 2610-2623.

[23] Li JN, Mahmoud MA, Han WF, Ripple M, Pizer ES. Sterol regulatory elementbinding protein-1 participates in the regulation of fatty acid synthase expression in colorectal neoplasia. Experimental Cell Research 2000;261:159-65.

[24] Swinnen JV, Vanderhoydonc F, Elgamal AA, Eelen M, Vercaeren I, Joniau S, et al. Selective activation of the fatty acid synthesis pathway in human prostate cancer. International Journal of Cancer. Journal international du cancer 2000;88:176-9.

[25] Yoon S, Lee MY, Park SW, Moon JS, Koh YK, Ahn YH, et al. Up-regulation of acetyl-CoA carboxylase alpha and fatty acid synthase by human epidermal growth factor receptor 2 at the translational level in breast cancer cells. The Journal of Biological Chemistry 2007;282:26122-31.

[26] Menendez JA, Lupu R. Fatty acid synthase and the lipogenic phenotype in cancer pathogenesis. Nature Reviews. Cancer 2007;7:763-77.

[27] Yellen P, Foster DA. Inhibition of fatty acid synthase induces pro-survival Akt and ERK signaling in K-Ras-driven cancer cells. Cancer Letters 2014;353(2): 258-263. http://dx.doi.org/10.1016/j.canlet.2014.07.027.

[28] Yahagi N, Shimano H, Matsuzaka T, Najima Y, Sekiya M, Nakagawa Y, et al. p53 Activation in adipocytes of obese mice. The Journal of Biological Chemistry 2003;278:25395-400.

[29] Assaily W, Rubinger DA, Wheaton K, Lin Y, Ma W, Xuan W, et al. ROSmediated p53 induction of Lpin1 regulates fatty acid oxidation in response to nutritional stress. Molecular Cell 2011;44:491-501.

[30] Liu Y, He Y, Jin A, Tikunov AP, Zhou L, Tollini LA, et al. Ribosomal proteinMdm2-p53 pathway coordinates nutrient stress with lipid metabolism by regulating $\mathrm{MCD}$ and promoting fatty acid oxidation. Proceedings of the National Academy of Sciences of the United States of America 2014;111:E2414-22.

[31] Kulawiec M, Ayyasamy V, Singh KK. p53 regulates mtDNA copy number and mitocheckpoint pathway. Journal of Carcinogenesis 2009;8:8.

[32] Bosch-Presegue L, Vaquero A. Sirtuins in stress response: guardians of the genome. Oncogene 2014;33(29):3764-75. http://dx.doi.org/10.1038/onc. 2013.344.

[33] Vaquero A, Sternglanz R, Reinberg D. NAD+-dependent deacetylation of H4 lysine 16 by class III HDACs. Oncogene 2007;26:5505-20.

[34] Vaquero A. The conserved role of sirtuins in chromatin regulation. The International Journal of Developmental Biology 2009;53:303-22.

[35] Blander G, Guarente L. The Sir2 family of protein deacetylases. Annual Review of Biochemistry 2004;73:417-35.

[36] Satoh A, Stein L, Imai S. The role of mammalian sirtuins in the regulation of metabolism, aging, and longevity. Handbook of Experimental Pharmacology $2011 ; 206: 125-62$

[37] Zhong L, Mostoslavsky R. Fine tuning our cellular factories: sirtuins in mitochondrial biology. Cell Metabolism 2011;13:621-6.

[38] Wellen KE, Thompson CB. A two-way street: reciprocal regulation of metabolism and signalling. Nature Reviews. Molecular Cell Biology 2012;13:270-6.

[39] DeBerardinis RJ, Lum JJ, Hatzivassiliou G, Thompson CB. The biology of cancer: metabolic reprogramming fuels cell growth and proliferation. Cell Metabolism 2008;7:11-20.

[40] Koppenol WH, Bounds PL, Dang CV. Otto Warburg's contributions to current concepts of cancer metabolism. Nature Reviews. Cancer 2011:11:325-37.

[41] Vander Heiden MG, Cantley LC, Thompson CB. Understanding the Warburg effect: the metabolic requirements of cell proliferation. Science 2009;324: 1029-1033.

[42] Metallo CM, Vander Heiden MG. Metabolism strikes back: metabolic flux regulates cell signaling. Genes \& Development 2010;24:2717-22.

[43] Haigis MC, Sinclair DA. Mammalian sirtuins: biological insights and disease relevance. Annual Review of Pathology 2010;5:253-95.

[44] Schwer B, Verdin E. Conserved metabolic regulatory functions of sirtuins. Cell Metabolism 2008;7:104-12

[45] Hallows WC, Lee S, Denu JM. Sirtuins deacetylate and activate mammalian acetyl-CoA synthetases. Proceedings of the National Academy of Sciences of the United States of America 2006;103:10230-35

[46] Kim SC, Sprung R, Chen Y, Xu Y, Ball H, Pei J, et al. Substrate and functional diversity of lysine acetylation revealed by a proteomics survey. Molecular Cell 2006;23:607-18

[47] Zhao S, Xu W, Jiang W, Yu W, Lin Y, Zhang T, et al. Regulation of cellular metabolism by protein lysine acetylation. Science 2010;327:1000-4.

[48] Lv L, Li D, Zhao D, Lin R, Chu Y, Zhang H, et al. Acetylation targets the M2 isoform of pyruvate kinase for degradation through chaperone-mediated autophagy and promotes tumor growth. Molecular Cell 2011;42:719-30.

[49] Cai L, Sutter BM, Li B, Tu BP. Acetyl-CoA induces cell growth and proliferation by promoting the acetylation of histones at growth genes. Molecular Cell 2011;42:426-37.

[50] Potapova IA, El-Maghrabi MR, Doronin SV, Benjamin WB. Phosphorylation of recombinant human ATP:citrate lyase by cAMP-dependent protein kinase abolishes homotropic allosteric regulation of the enzyme by citrate and increases the enzyme activity. Allosteric activation of ATP:citrate lyase by phosphorylated sugars. Biochemistry 2000;39:1169-79.

[51] Berwick DC, Hers I, Heesom KJ, Moule SK, Tavare JM. The identification of ATP-citrate lyase as a protein kinase B (Akt) substrate in primary adipocytes. The Journal of Biological Chemistry 2002;277:33895-900.

[52] Sale EM, Hodgkinson CP, Jones NP, Sale GJ. A new strategy for studying protein kinase B and its three isoforms. Role of protein kinase B in phosphorylating glycogen synthase kinase-3, tuberin, WNK1, and ATP citrate lyase. Biochemistry 2006;45:213-23. 
[53] Luna A, Aladjem MI, Kohn KW. SIRT1/PARP1 crosstalk: connecting DNA damage and metabolism. Genome Integrity 2013:4:6.

[54] Flick F, Luscher B. Regulation of sirtuin function by posttranslational modifications. Frontiers in Pharmacology 2012;3:29.

[55] Nasrin N, Kaushik VK, Fortier E, Wall D, Pearson KJ, de Cabo R, et al. JNK1 phosphorylates SIRT1 and promotes its enzymatic activity. PLoS ONE 2009;4:e8414.

[56] Lee CW, Wong LL, Tse EY, Liu HF, Leong VY, Lee JM, et al. AMPK promotes p53 acetylation via phosphorylation and inactivation of SIRT1 in liver cancer cells. Cancer Research 2012;72:4394-404.

[57] Lau AW, Liu P, Inuzuka H, Gao D. SIRT1 phosphorylation by AMP-activated protein kinase regulates p53 acetylation. American Journal of Cancer Research 2014;4:245-55.

[58] Kang H, Jung JW, Kim MK, Chung JH. CK2 is the regulator of SIRT1 substratebinding affinity, deacetylase activity and cellular response to DNA-damage. PLoS ONE 2009;4:e6611.

[59] Zschoernig B, Mahlknecht U. Carboxy-terminal phosphorylation of SIRT1 by protein kinase CK2. Biochemical and Biophysical Research Communications 2009;381:372-7.

[60] Kang H, Suh JY, Jung YS, Jung JW, Kim MK, Chung JH. Peptide switch is essential for Sirt1 deacetylase activity. Molecular Cell 2011;44:203-13.

[61] Sasaki T, Maier B, Koclega KD, Chruszcz M, Gluba W, Stukenberg PT, et al. Phosphorylation regulates SIRT1 function. PLoS ONE 2008;3:e4020.

[62] Yi J, Luo J. SIRT1 and p53, effect on cancer, senescence and beyond. Biochimica et Biophysica Acta 2010;1804:1684-9.

[63] Langley E, Pearson M, Faretta M, Bauer UM, Frye RA, Minucci S, et al. Human SIR2 deacetylates p53 and antagonizes PML/p53-induced cellular senescence. The EMBO Journal 2002;21:2383-96.

[64] Canto C, Auwerx J. Interference between PARPs and SIRT1: a novel approach to healthy ageing. Aging 2011;3:543-7.

[65] Lowe SW, Lin AW. Apoptosis in cancer. Carcinogenesis 2000;21:485-95.

[66] Cheng HL, Mostoslavsky R, Saito S, Manis JP, Gu Y, Patel P, et al. Developmental defects and p53 hyperacetylation in Sir2 homolog (SIRT1)-deficient mice. Proceedings of the National Academy of Sciences of the United States of America 2003;100:10794-99.

[67] Vogelstein B, Lane D, Levine AJ. Surfing the p53 network. Nature 2000;408:307-10.

[68] Han MK, Song EK, Guo Y, Ou X, Mantel C, Broxmeyer HE. SIRT1 regulates apoptosis and Nanog expression in mouse embryonic stem cells by controlling p53 subcellular localization. Cell Stem Cell 2008;2:241-51.

[69] Brooks CL, Gu W. How does SIRT1 affect metabolism, senescence and cancer Nature Reviews. Cancer 2009;9:123-8.

[70] Deng CX. SIRT1, is it a tumor promoter or tumor suppressor. International Journal of Biological Sciences 2009:5:147-52.

[71] Houtkooper RH, Pirinen E, Auwerx J. Sirtuins as regulators of metabolism and healthspan. Nature Reviews. Molecular Cell Biology 2012;13:225-38.

[72] Saunders LR, Verdin E. Sirtuins: critical regulators at the crossroads between cancer and aging. Oncogene 2007:26:5489-504.

[73] van Gent R, Di Sanza C, van den Broek NJ, Fleskens V, Veenstra A, Stout GJ, et al. SIRT1 mediates FOXA2 breakdown by deacetylation in a nutrientdependent manner. PLoS ONE 2014:9:e98438.

[74] Yeung F, Ramsey CS, Popko-Scibor AE, Allison DF, Gray LG, Shin M, et al. Regulation of the mitogen-activated protein kinase kinase (MEK)- 1 by NAD-dependent deacetylases. Oncogene 2014. http://dx.doi.org/10.1038/ onc.2014.39.

[75] Ramakrishnan G, Davaakhuu G, Kaplun L, Chung WC, Rana A, Atfi A, et al. Sirt2 deacetylase is a novel AKT binding partner critical for AKT activation by insulin. The Journal of Biological Chemistry 2014;289:6054-66.

[76] Rabadi MM, Xavier S, Vasko R, Kaur K, Goligorksy MS, Ratliff BB. Highmobility group box 1 is a novel deacetylation target of Sirtuin1. Kidney International 2014. http://dx.doi.org/10.1038/ki.2014.217.

[77] Wang RH, Zheng Y, Kim HS, Xu X, Cao L, Luhasen T, et al. Interplay among BRCA1, SIRT1, and Survivin during BRCA1-associated tumorigenesis. Molecular Cell 2008;32:11-20.

[78] Roth M, Chen WY. Sorting out functions of sirtuins in cancer. Oncogene 2014;33:1609-20.

[79] Yao Y, Yang Y, Zhu WG. Sirtuins: nodes connecting aging, metabolism and tumorigenesis. Current Pharmaceutical Design 2014;20:1614-24.

[80] Luo J, Nikolaev AY, Imai S, Chen D, Su F, Shiloh A, et al. Negative control of p53 by Sir2alpha promotes cell survival under stress. Cell 2001;107:137-48.

[81] Vaziri H, Dessain SK, Ng Eaton E, Imai SI, Frye RA, Pandita TK, et al. hSIR2(SIRT1) functions as an NAD-dependent p53 deacetylase. Cell 2001:107:149-59.

[82] Vaquero A, Scher M, Lee D, Erdjument-Bromage H, Tempst P, Reinberg D. Human SirT1 interacts with histone $\mathrm{H} 1$ and promotes formation of facultative heterochromatin. Molecular Cell 2004;16:93-105.

[83] Bosch-Presegue L, Raurell-Vila H, Marazuela-Duque A, Kane-Goldsmith N, Valle A, Oliver J, et al. Stabilization of Suv39H1 by SirT1 is part of oxidative stress response and ensures genome protection. Molecular Cell 2011;42:210-23.

[84] O'Hagan HM, Mohammad HP, Baylin SB. Double strand breaks can initiate gene silencing and SIRT1-dependent onset of DNA methylation in an exogenous promoter CpG island. PLoS Genetics 2008;4:e1000155.

[85] Pruitt K, Zinn RL, Ohm JE, McGarvey KM, Kang SH, Watkins DN, et al. Inhibition of SIRT1 reactivates silenced cancer genes without loss of promoter DNA hypermethylation. PLoS Genetics 2006;2:e40.

[86] Fan W, Luo J. SIRT1 regulates UV-induced DNA repair through deacetylating XPA. Molecular Cell 2010;39:247-58.
[87] Ming M, Shea CR, Guo X, Li X, Soltani K, Han W, et al. Regulation of global genome nucleotide excision repair by SIRT1 through xeroderma pigmentosum C. Proceedings of the National Academy of Sciences of the United States of America 2010;107:22623-28.

[88] Gao Y, Ferguson DO, Xie W, Manis JP, Sekiguchi J, Frank KM, et al. Interplay of p53 and DNA-repair protein XRCC4 in tumorigenesis, genomic stability and development. Nature 2000;404:897-900.

[89] Jeong J, Juhn K, Lee H, Kim SH, Min BH, Lee KM, et al. SIRT1 promotes DNA repair activity and deacetylation of Ku70. Experimental \& Molecular Medicine 2007;39:8-13.

[90] Li K, Casta A, Wang R, Lozada E, Fan W, Kane S, et al. Regulation of WRN protein cellular localization and enzymatic activities by SIRT1-mediated deacetylation. The Journal of Biological Chemistry 2008;283:7590-8.

[91] Yuan Z, Seto E. A functional link between SIRT1 deacetylase and NBS1 in DNA damage response. Cell Cycle 2007;6:2869-71.

[92] Yuan Z, Zhang X, Sengupta N, Lane WS, Seto E. SIRT1 regulates the function of the Nijmegen breakage syndrome protein. Molecular Cell 2007;27:149-62.

[93] Wang RH, Sengupta K, Li C, Kim HS, Cao L, Xiao C, et al. Impaired DNA damage response, genome instability, and tumorigenesis in SIRT1 mutant mice. Cancer Cell 2008;14:312-23.

[94] Oberdoerffer P, Michan S, McVay M, Mostoslavsky R, Vann J, Park SK, et al. SIRT1 redistribution on chromatin promotes genomic stability but alters gene expression during aging. Cell 2008;135:907-18.

[95] Gupta A, Guerin-Peyrou TG, Sharma GG, Park C, Agarwal M, Ganju RK, et al. The mammalian ortholog of Drosophila MOF that acetylates histone H4 lysine 16 is essential for embryogenesis and oncogenesis. Molecular and Cellular Biology 2008;28:397-409.

[96] Rea S, Xouri G, Akhtar A. Males absent on the first (MOF): from flies to humans. Oncogene 2007;26:5385-94.

[97] Ikura T, Ogryzko VV, Grigoriev M, Groisman R, Wang J, Horikoshi M, et al. Involvement of the TIP60 histone acetylase complex in DNA repair and apoptosis. Cell 2000;102:463-73.

[98] Peng L, Ling H, Yuan Z, Fang B, Bloom G, Fukasawa K, et al. SIRT1 negatively regulates the activities, functions, and protein levels of hMOF and TIP60. Molecular and Cellular Biology 2012;32:2823-36.

[99] Sun Y, Jiang X, Chen S, Fernandes N, Price BD. A role for the Tip60 histone acetyltransferase in the acetylation and activation of ATM. Proceedings of the National Academy of Sciences of the United States of America 2005;102:13182-87.

[100] Sawada M, Sun W, Hayes P, Leskov K, Boothman DA, Matsuyama S. Ku70 suppresses the apoptotic translocation of Bax to mitochondria. Nature Cell Biology 2003:5:320-9.

[101] Cohen HY, Lavu S, Bitterman KJ, Hekking B, Imahiyerobo TA, Miller C, et al. Acetylation of the $C$ terminus of Ku70 by CBP and PCAF controls Baxmediated apoptosis. Molecular Cell 2004;13:627-38.

[102] Cohen HY, Miller C, Bitterman KJ, Wall NR, Hekking B, Kessler B, et al. Calorie restriction promotes mammalian cell survival by inducing the SIRT1 deacetylase. Science 2004;305:390-2.

[103] McBurney MW, Yang X, Jardine K, Bieman M, Th'ng J, Lemieux M. The absence of SIR2alpha protein has no effect on global gene silencing in mouse embryonic stem cells. Molecular Cancer Research: MCR 2003;1:402-9.

[104] McBurney MW, Yang X, Jardine K, Hixon M, Boekelheide K, Webb JR, et al. The mammalian SIR2alpha protein has a role in embryogenesis and gametogenesis. Molecular and Cellular Biology 2003;23:38-54.

[105] Li T, Kon N, Jiang L, Tan M, Ludwig T, Zhao Y, et al. Tumor suppression in the absence of p53-mediated cell-cycle arrest, apoptosis, and senescence. Cell 2012;149:1269-83.

[106] Su X, Gi YJ, Chakravarti D, Chan IL, Zhang A, Xia X, et al. TAp63 is a master transcriptional regulator of lipid and glucose metabolism. Cell Metabolism 2012;16:511-25.

[107] Rufini A, Niklison-Chirou MV, Inoue S, Tomasini R, Harris IS, Marino A, et al. TAp73 depletion accelerates aging through metabolic dysregulation. Genes \& Development 2012;26:2009-14.

[108] Berkers CR, Maddocks OD, Cheung EC, Mor I, Vousden KH. Metabolic regulation by 53 family members. Cell Metabolism 2013;18:617-33.

[109] von Stechow L, Ruiz-Aracama A, van de Water B, Peijnenburg A, Danen E Lommen A. Identification of cisplatin-regulated metabolic pathways in pluripotent stem cells. PLoS ONE 2013;8:e76476.

[110] Lukas J, Lukas C, Bartek J. More than just a focus: the chromatin response to DNA damage and its role in genome integrity maintenance. Nature Cell Biology 2011;13:1161-9.

[111] Shi L, Oberdoerffer P. Chromatin dynamics in DNA double-strand break repair. Biochimica et Biophysica Acta 2012:1819:811-9.

[112] Soria G, Polo SE, Almouzni G. Prime, repair, restore: the active role of chromatin in the DNA damage response. Molecular Cell 2012;46:722-34.

[113] Gonfloni S. Targeting DNA damage response: threshold, chromatin landscape and beyond. Pharmacology \& Therapeutics 2013;138:46-52.

[114] Smeenk G, van Attikum H. The chromatin response to DNA breaks: leaving a mark on genome integrity. Annual Review of Biochemistry 2013;82:55-80.

[115] Liu J, Kim J, Oberdoerffer P. Metabolic modulation of chromatin: implications for DNA repair and genomic integrity. Frontiers in Genetics 2013;4:182.

[116] Kruse JP, Gu W. Modes of p53 regulation. Cell 2009;137:609-22.

[117] Comel A, Sorrentino G, Capaci V, Del Sal G. The cytoplasmic side of p53's oncosuppressive activities. FEBS Letters 2014;588(16):2600-9. http:/ dx.doi.org/10.1016/j.febslet.2014.04.015.

[118] Jiang P, Du W, Yang X. p53 and regulation of tumor metabolism. Journal of Carcinogenesis 2013;12:21. 